\title{
Role of Computed Tomography (CT) in diagnosis of urgent head trauma in adult
}

\author{
Adil Ismail, Eenam Azez \\ Department of Radiology Techniques, Health and Medical Technology, Baghdad, Iraq
}

\begin{abstract}
Background. Head injury is one of the leading causes of morbidity and mortality throughout the world. Fall injury is the main cause of head trauma followed by road traffic accidents (RTA) and physical assault. Every victim with altered level of consciousness must be evaluated for brain injury. Radiological evaluation has undergone dramatic changes with the advent of computed tomography $(\mathrm{CT})$ as it can precisely define the nature and location of the culprit lesion(s).

Aim and projective. The aims of this study are to identify the major CT scan findings in suspected cases of Head injury.

Materials and methods. This is an exploratory study carried out in the specialized surgical hospital and neurosurgical hospital from October 2016 to July 2017 on patients attending to the emergency department with acute head trauma and examined by spiral computed tomography (CT). All patients undergo a full neurological examination.

Result. The study was conducted on 160 patients, 106 males and 54 females, by males: females = 2: 1, aged 21-64 years, with an average age of 43 years.

One hundred twenty patients $(75 \%)$ had abnormal CT findings, while 40 patients $(25 \%)$ had normal CT.

Patients with blunt (closed) head injury were 126 (78.75\%), while 34 patients (21.25\%) had penetrating (open) head injury.

In this study, the number of patients was $160,106(66 \%)$ of males and $54(34 \%)$ of females with male to female ratio 2/1. The most common age group was between 20-29 years as shown in (Table 1 and Fig. 1).

The patient had severe (closed) head injuries (16\%), while 34 patients $(21.25 \%)$ had an open head injury. Data analysis was performed in this study as shown in Table 2 and Fig. 2 are frequency, consciousness level disorders were found in 62 patients (38.75\%), followed by multiple injuries including chest and abdominal injuries in 34 patients (21.2), injury penetration Head in 31 patients (19.3\%). After a head injury in 30 patients $(18.7 \%)$, vomiting over one episode was seen in 17 patients $(10.6 \%)$, and only 6 patients $(3.7 \%)$ were provided with signs of fracture at the base of the skull including runny nose Warm CSF, vessels, skull double nerve, battle mark (Mastoid) and raccoon (black) eye.

Twenty-one patients (75\%) had abnormal CT scan results while 40 (25\%) had regular CT as shown in (Table 3 and Fig. 3).

CT abnormalities are shown in accordance with the mechanism of infection (Table 3 and Fig. 3), indicating that the swelling of the entire brain occurs mainly in adolescents and children, and the most age group between 20-29 years as shown in Table 1 and Fig. 1), The patient with severe injuries was 16\% (10\%), while 34 patients (21.25\%) had an open head injury.

CT resection of the mechanism is less in (Table 3 and Fig. 3), indicating that the brain swelling. There are

Road traffic accident (RTA) was the most common cause of acute head trauma seen in 63 patients (39.4\%), followed by penetrating injury seen in 34 patient $(21.2 \%)$, fall from height in 28 patients (17.5\%) blunt injury of blast in 25 patient $(15.7 \%)$ and assault seen in 10 patient $(6.2 \%)$.

Acute head trauma was divided into 3 types according to the Glasgow coma scale (GCS) score including: moderate head trauma (score 9-12) was the commonest type seen in 60 patients $(37.5 \%), 48(80 \%)$ of them had abnormal CT findings, mild head trauma (score 13-15) seen in 53 patients (33.2\%), $33(62.2 \%)$ of them had abnormal CT, and severe head trauma (score $<8$ ) seen in 47 patients $(29.3 \%), 39(83 \%)$ of them had abnormal CT findings.

Skull fracture was the commonest abnormal CT finding, seen in 70 patients $(43.7 \%)$,followed in order of frequency by: cerebral contusion in 47 patients $(29.3 \%)$, subgalial hematoma in 34 patients $(21.2 \%)$, intracranial air seen in 27 patients $(16.8 \%)$, epidural hematoma in 15 patients $(9.3 \%)$, subdural hematoma seen in 12 patients $(7.5 \%)$ subarachnoid hemorrhage in 11 patients $(6.8 \%)$, diffuse cerebral edema in 10 patients $(6.2 \%)$, and intraventricular hemorrhage seen in 8 patients $(5 \%)$.

Eighty-eight patients $(55 \%)$ had skull x-ray, $(62.5 \%)$ of them were normal, $(21.5 \%)$ had linear skull fracture, $(9.2 \%)$ had comminuted fracture and $(6.8 \%)$ had depressed fracture.

The main indication for CT scan was disturbance in the level of consciousness and was found in 62 patients (38.7\%).
\end{abstract}




\begin{abstract}
Conclusion. We conclude that CT is now the first imaging technique used after acute head trauma in those places. Where CT scans are available. CT is the preferred imaging technique for assessing head trauma. CT was found highly sensitive in demonstrating fractures and was considered the modality of choice when imaging suspected cases of head injury and in emergency patients due to its accuracy, reliability, safety and wide availability.

CT scan is still the primary method for evaluating patients with acute head trauma, which is fast, easy and reliable, and is the best choice for cranial fractures and acute axial or acute intraocular bleeding.

$\mathrm{CT}$ is the imaging method chosen in the evaluation of patients with acute injuries. CT is valuable in making film diagnosis, as well as excluding alternative diagnosis, or crevice of other pathologies, even among uncooperative patients. Patient monitoring is safe and simple; CT is compatible with other patient install devices. The identity and localization of cranial fracture and bone/metal fragment are easily determined. Evaluation of acute bleeding and mass effects is optimal.
\end{abstract}

Keywords: CT scan, Head Trauma, scale (GCS), Hematoma

\section{INTRODUCTION}

Head trauma it is any injury that causes damage or functional disturbance to the cranium, meninges and brain (Bordignon and Arruda, 2002) this agreement with the results obtain by author (Jenett, 1996).

CT presented for the first time by Godfrey Hounsfield in 1972, open new opportunities in the investigation of head injury and is now the investigation of choice (Shehu, 2002). Then He gave a Nobel Prize lecture for this landmark discovery in 1979 and noted at the time. Our view of the body is separated by a chain of known (1).

A CT scanner can make these slices thin or imported on a part of the body, being 2 to $10 \mathrm{~m}$ bullets, and it takes (2). Assessment of patients with intracranial injury or head trauma, one of the key issues to be addressed is whether the patient should be investigated using imaging to confirm or refute the presence of an injury (epilepsy) that would alter subsequent medical treatment or investigation of Patient. When trying to decide on the use or nonuse of diagnostic imaging, it is important to focus on the features of history and physical examination that have been found to be associated with clinically significant intracranial injury. At first, it may seem simpler, faster and safer to simply perform a imaging test on anyone with a head trauma.

\section{Head trauma}

Head trauma is found in all age groups, particularly among young people, whose peak is between the ages of 18 and 30, Road accidents (RTA) are the leading cause of head health in the general population, especially the young age group and represents about $50 \%$ of all traumatic brain injuries Waterfalls are the second leading cause of brain injuries that occur from 20 to $30 \%$ of all brain injuries. Fashion Mechanics, Road accidents (RTA) had been shown to be the most important causes of head injury in several represented as was reported by (Abajimi et al., 2002).

From a traumatic shock, it needs rapid and non-effective evaluation (4).

The painful fingers of the brain can generally be divided into two groups, closed head injuries and head injuries. By definition, the Dura is intact in injuries (5).

She went out of her way. Some symptoms are obvious immediately, while others are affected. People with mild head trauma may eat in consciousness or lose consciousness for a few minutes or seconds. Some people may also be dazed or not. People with mild or solid shocks or inability to grow, change, be affected or think (5).

People suffering from psychological or compulsive trauma or psychological distress weakness or numbness in the limbs, tampering with speech, loss of coordination, and/or increased confusion to restlessness or agitation (5).

CT was first introduced by Godfrey Hounsfield in 1972. He delivered his Nobel prize lecture for this outstanding discovery in 1979 and noted at that time; we looked at the body separated in to a series of thin slices (1). The CT scanner can make these slices as thin or thick as desired, but usually, depending on the body part, the slices are 2 to 10 millimeters thickness, it takes about 12 to 16 slices to look at the entire brain. (3) Head trauma account for a substantial proportion of morbidity or mortality in all age group, particularly affecting young population with peak age group between 18 -30 years $(2,4)$.

The males are approximately twice as likely as females sustain traumatic brain injury (8). 
Road traffic Accident (RTA) is the leading cause of head trauma in the general population, especially young age group and account for approximately $50 \%$ of all traumatic brain injuries. Falls are the second leading cause of traumatic brain injuries account for $20-30 \%$ OF all traumatic brain injuries. CT has given a forward push forward to intracranial imaging in general, and the diagnosis of subtypes of CVA in particular. Other mechanisms of acute head trauma in adults include: penetrating injury and blast injuries (Obajimi et al. 2002).

For management and triage of people with cranial trauma, rapid accurate and noninvasive assessment is required (6). Traumatic brain injuries can be generally divided into two groups, closed head injuries and open head injuries. By definition, dura is intact in closed head injuries, in comparison with open traumas in which dura is torn (7). Symptoms of traumatic brain Injuries may be mild, moderate or severe, depending on how the damage is extended to the brain. Some symptoms are evident immediately, while others do not appear until many days or weeks after the injury. People with mild head traumas may remain conscious or experience loss of consciousness for a few minutes or seconds. Some people may also fell dazed or not like themselves for many days or weeks following the initial injury.

People with mild head trauma may show other symptoms such as confusion, dizziness, headache, blurred vision or tired eyes, fatigue or lethargy, bad taste in the mouth, changing of sleep patterns, behavioral or mood changes, memory trouble, loss of concentration, attention or thinking (5).

People with moderate or severe head traumas may show the same above symptoms, but they can also develop headache which gets worse or does not go away, nausea or repeated vomiting, seizures or convulsions, difficulty to awaken from sleep, dilatation of one or both pupils of eyes, weakness or numbness in the extremities, slurred speech, loss of coordination, and/or increased confusion as well as restlessness or agitation (8).

\section{Glasgow COMA Scale (GCS)}

In 1974, the GCS was introduced to serve as a clinical standard for the assessment of the severity of traumatic brain injury since that time, it has also been adopted by clinician as a means of assisting in long term outcome prediction after traumatic brain injury, The GCS is intended to represent a numeric expression of the clinical severity of traumatic brain injury (9).

Eye opening

- Spontaneous $=4$

- To speech $=3$

- Painful stimulation $=2$

- No response $=1$

Motor response

- Follows commands $=6$

- Make localizing movement to pain $=5$

- Make withdrawal movement to pain $=4$

- Flexor (decorticate) posturing to pain $=3$

- Extensor (decorticate) posturing to pain $=2$

- No response $=1$

Verbal response

* Oriented $=5$

* Confused $=4$

* Says inappropriate words $=3$

* Says incomprehensible sounds $=2$

* No response $=1$

The severity of traumatic brain injury according to the GCS score is as follows; (10)

- Mild head injury; score 13-15

- Moderate head injury; score 9-12

- Severe head injury; score $\geq 8$

\section{Diagnostic Imaging in head trauma}

\section{Conventional Radiography}

It is not possible to detect each linear fracture in the basement in a routine radiographic examination of the skull. The determination of fracture depends on radiography on the width and direction of the fracture. Although simple x-rays can reveal skull fractures, they are now obsolete. Computed tomography with a bone window is more accurate in detecting fractured skull fractures and more sensitive than cranial radiography. (Pfeiffer and Bab 2010) reviewed several studies of lost injuries in trauma patients and noted that the incorrectly interpreted $\mathrm{x}$-rays (15-34.9\%) were major radiological factors contributing to the lost diagnosis. Lack of clinical experience (26.5\%), evaluation errors (33.8$60.5 \%$ ), and technical errors are additional contributing factors. The cranial stitches and fractures are mixed several times in x-rays. It may also be difficult to distinguish vascular markers from fractions (11). 


\section{Computed Tomography Scanning (CT)}

CT scan is the imaging method of choice in the assessment of acutely injured patients. It is quick, easy, reliable and viable procedure. $\mathrm{CT}$ is valuable in making film diagnosis, as well as excluding alternative diagnosis, or the squeal of other pathology, even among uncooperative patients. Patient monitoring is safe \& simple safe, and CT is compatible with other patient stabilization devices. Identification and localization of Calvary fracture and a bony/metallic fragment are easily achieved. Assessment of acute hemorrhages and mass effects is optimal (12). CT scan with intravenous contrast is usually unnecessary; furthermore, contrast infusion imaging may obscure some pathology in the acute setting (6).

\section{Magnetic Resonance Image (MRI)}

Almost all studies on head trauma have used the CT scanner as a gold standard for the detection of intracranial abnormalities, but MRI has been shown to be up to five times more sense for detecting non hemorrhagic lesions (e.g. Contusion and diffused axonal injury). This is particularly true for lesions in the skull base, the posterior fossa and in fine non hemorrhagic lesions (Mittle et al1994) this agreement with the results obtain by author (Sang Won Chung at el.2012) reported that $30 \%$ of patients with a negative head CT have radiographic evidence of diffuse axonal injury on MRI $(13,14,15)$.

\section{Conventional Catheter Angiography}

Depending on practice patterns at individual centers, conventional catheter angiography is considered in patients with penetrating trauma, parenchymal and subarachnoid hemorrhage, both as diagnostic test as it may show active bleeding from a ruptured artery and traumatic aneurysm and as a treatment option, but it has long been superseded by noninvasive imaging especially $\mathrm{CT}$ and MRI $(16,17)$.

\section{Types of injury}

Primary injuries

\section{Skull fracture}

Skull fractures present in approximately twothirds of patients with acute head injury. Skull fractures may involve the caldarium (VAULT) and the skull base and these may be simple or complex and include; linear, depressed, diastolic, comminuted and compound types $(10,18)$.

Linear fractures are the most common of all fractures, and are readily recognized on good quality skull X-ray film, where they are often shown as linear lucent lines. High resolution CT may be able to detect linear skull fractures, however, it is more difficult on CT than with skull radiography to distinguish cranial suture from linear fractures $(18,19)$.

Depressed fractures usually result from high velocity injuries, in skull radiographs these may appear as cranial injuries (Gardner, et al, 2011) this agreement with the results obtain by author (A. Agrawa, 2019) areas of increased density due to an overlapping of bone margins, but $\mathrm{CT}$ is better than skull radiographs in demonstrating depressed fractures provided bone windows are used, because without use of bone window a fractures may be confused epidural hematoma, CT is also capable of demonstrating the depth of depressed fracture and any accompanying intracranial injuries (18).

Comminuted fracture by definition refers to fracture consisting of multiple bone fragments. Most depressed skull fracture is comminuted. Compound fracture is also known as the open fracture, this type of fracture communicates directly with the scalp, skull base, or Para nasal sinuses and most commonly caused by penetrating injury. Comminuted fractures are also compound fractures $(10,18)$.

Diastolic fractures are common in children and should 12 be differentiated from normal cranial sutures, unilateral widening of paired cranial sutures may be considered as evidence of diastasis and often associated with bony fragments within the suture (11).

CT may replace the need for tangential skull radiography for depressed, comminuted, compound or even diastolic fractures. The top of the vertex may be over looked by axial CT examination yet it can be easily demonstrated by coronal CT examination (18).

Basilar skull fracture although not generally considered a separate type of skull fracture. The Basilar skull fracture is a rather distinct form of fracture, it's also consist of any or all of the types of fracture defined previously, including linear, depressed, diastolic, comminuted an compound. 
Basilar skull fractures are often extremely difficult to identify on plain radiograph, they may, however, be suspected clinically as a result of symptoms such as CSF, rhinorrhea, otorrhea, hemotypanum and cranial nerve dysfunction. Basilar skull fracture are best diagnosed by high resolution thin section CT of the skull base and are commonly accompanied by air fluid levels in the sinuses or middle ear cavity $(10,18)$.

\section{Extra cranial Hematoma}

Scalp swelling is the rule rather than the exception in significant head injury, and may be the clue to an underlying fracture. On CT this often indicates the point of impact, and contusion is often found in the surface of the brain diametrically opposite (17).

\section{Epidural hematoma}

The Dura mater consist of 2 closely invested with each other layers, the visceral or meningeal layer, which lines the intracranial space, and the parietal layer, which functions as the periosteal of the caldarium. The Dura therefore, adheres tightly to the cranial sutures (16).

Skull fractures that cross arterial branches may bleed into the closed space between the caldarium and the periosteal layer forming homogenously high density lens-shaped or biconvex lesions in CT termed epidural hematomas, which are most commonly of arterial type due to the damage of the middle meningeal arteries. In majority of cases, epidural hematomas are associated with skull fractures, and they are most frequent in the front parietal regions $(15,19,21)$.

Venous epidural hematomas happen when fractures disrupt one of the sinuses, and they usually happen in the posterior fossa, when they may be particularly missed if poor quality CT scanner is used. Due to the delayed bleeding onset after trauma, this type of epidural hematoma usually requires delayed imaging $(11,19)$.

\section{Subdural hematoma}

The subdural space is bound externally by the meningeal layer of the Dura and externally by the arachnoid mater. Haematoma confined by the reflected Dura of tentorium and falx but easily spread in to intervening potential space to form crescenticor convex-out/concave-in collection called subdural haematomas. Tearing of the birding cortical veins that Travers this space accounts for most subdural hematoma. Usually from abrupt acceleration/ deceralation injury. This is more likely to happen in old age patients and other patients with atrophy, where subdural space is enlarged $(20,21)$.

In CT, acute subdural hematomas have higher attenuation than brain for about 2 weeks, and after 3-4 weeks, they will have lower attenuation, eventually approaching that of CSF. Between 2-4 weeks after injury, they pass through a stage when they are in Odense with the underlying brain that is called is Odense hematoma, which can cause major problems in diagnosis, since the lesion is not visible directly, and it may be useful to depend upon the indirect signs including ventricular or pineal displacement, and absence of visible sulci on the affected side. Intravenous iodinated contrast medium can define the collection by induction of the enhancement of the hematoma's capsule $(19,22)$.

In contradistinction to epidural hematomas, there is no consistent relationship to the presences of skull fractures, and subdural hematoma can cross suture margins but nodular attachments. Most of subdural hematomas are supratentorial, located along the convexity (11).

In the follow up and management of both extra \& subdural hematomas, CT scanning is considered as an invaluable procedure, because it permits to detect residual or recurrent lesions of undiagnosed contralateral hematomas or even the presence of an infection. Infection may be evidenced by presence of lobulated air within the hematoma cavity, by increase of the fluid content density, and by marked capsular enhancement. The calcification of capsules may occur in chronic lesions, with or without complications as seen in infections (19).

\section{Subarachnoid Hemorrhage}

Subarachnoid space extends between the arachnoid matter and the cortical/pia matter surface of the brain (20).

Subarachnoid Hemorrhage results from disruption of small suarachnoidal vessels or direct extension in to the subarachnoid space by a contusion or hematomas, it most commonly follows severe head injury $(7,17)$. 
Subarachnoid Hemorrhage results in diffusion of blood diluted with CSF throughout the sub arachnoid space of the cortical sulci or cisterns. In CT it appears as increased density of the basal cisterns, the Sylvain fissure, the interhemspheric fissure, and the tentorium, yet it may be is Odense if there is insufficient blood. Subarachnoid hemorrhage in the interhemispheric fissure appears as ahyperdense line and should be distinguished from (18,23).

\section{Intraventicular Hemorrhage}

Intraventicular Hemorrhage results from rotationally induced tearing of subepindymal veins on the surface of the ventricles or by direct extension of parenchymal hematoma in to ventricular system. Even in severe head injury interventicular hemorrhage is unusual (3\%) most common occurring when an itracerebral hemorrhagic contusion or hemato0ma breaks into the ventricle $(7,24)$.

On CT interventicular hemorrhage appears as increase attenuation within the ventricular system that may be accompanied by a blood fluid layer. In the absence of blood fluid layer, the overall density of the ventricles may be increased significantly to the extent that all CSF within the ventricular system appear white. It is more often that the CSF within ventricles appears of increased density compared with the normal CSF attenuation or blood identified as layering within the ventricles (10).

\section{Cerebral Contusion}

Cerebral Contusion is bruises and laceration of the brain which are covered by Dura. These may occur either at the site of impact (coup contusion) or at the opposite side as brain rebounds (countercoup contusion). There are two types of cerebral contusion which can be detected by CT scanning, the hemorrhagic and non-hemorrhagic contusions $(7,21)$.

Hemorrhagic contusion commonly seen in inferior frontal and anterior temporal lobes and bleeding is often subcortical. In CT scan it appears as amass lesion of mixed high and low density with narrow rim of surrounding edema. The hemorrhagic area may not be observed in the very acute stage; they occur within 24 hours or later $(19,24)$.

Non hemorrhagic contusion cannot reliably be distinguished from cerebral edema, but tend to be more focal and space occupying .In intravenous contrast medium, considerable enhancement may be seen, and this does not usually occur with edema (19).

\section{Diffuse brain damage}

No obvious focal lesions or masses are seen in about $50 \%$ of patient suffering from immediate prolonged unconsciousness after head injuries, and this is true in about one third of fatal classes. In those patients, the lesions have been classified into:

\section{Multiple petechial hemorrhages}

This type of injury which is seen within the brainstem and white matter is rapidly fatal. Therefore, it is more likely to be seen at postmortem than in imaging departments (19).

Diffuse anoxal injury (white matter shearing).

It's one of the most types of primary neuronal injury in a patient with severe head trauma. It is characterized by a widespread disruption of axons that occurs at the time of an acceleration or deceleration injury. Patients are unconscious from the time of impact and remain unconscious or severely disabled until death $(7,19)$. The characteristic site of diffuse axonal is gray matter/white matter junction in parasagittal areas, corpus callosum and dorsolateral brain stem (11).

Little or nothing may be shown at imaging, despite the severe damage of brain and the very grave state of patients. Initial CT is often normal and MRI is more sensitive in its detection especially in susceptibility (T2) MRI $(7,19)$.

\section{Brain swelling}

Diffuse swelling of entire brain takes place mainly in adolescents and children. It's of several types in head injury, and the commonest is hyperemic brain swelling, where the brain swells due to an increase in blood volume, not edema. Greywhite matter contrast is preserved on both CT and MRI and it can be difficult to assess $(17,19)$.

With focal \& mass lesions, diffuse swelling of the ipsilateral hemisphere may also happen. This includes acute subdural hematoma \& extensive unilateral contusion (19).

Diffuse bilateral swellings in the ventricles are seen as slit-like compressed and small at imaging. 
The basal cistern may be occluded \& the sulci effacedematouscer. The cerebellum may appear relatively hyper dense in comparison with edematous cerebral hemispheres (white cerebral sign). At CT scanning, and in the early stage, no reduction in the white matter's density is shown and appears normal, despite the evidence of edema in prolonged coma cases $(7,19)$.

\section{Hypoxic brain damage}

It occurs due to either arterial spasm or prolonged decrease in the systolic blood pressure or to both. Hypoxia and subsequent infarctions can also be the result of brain swelling and tentoria herniation leading to compromise the posterior cerebral circulation, or it can be a result of trauma to major vessels. In the early stages, imaging may show little, but evidence of infarction is demonstrated later (19).

\section{Intracranial air}

Intracranial air implies a dural tear communicating with sinuses or other air containing cavity. Intracerebral, subdural or subarachnoid air is readily recognized at $\mathrm{CT}$, which is more sensitive than plain radiography in small air quantities recognition (19).

\section{Penetrating injury}

CT is the method of choice to diagnose patients with gunshot wound and other penetrating traumas. The course of projectile may be identified, and the location of relevant foreign body and its effects on intracranial strictures can easily be assessed. However, good demonstration of the relationship of metallic objects may be prevented by the resulting artifacts. Wooden fragments may appear less dense than brain... $(2,19)$.

\section{Brain stem injury}

Brain stem injury is the gravest intracranial lesions, with very high mortality and morbidity (4).

The most common form of primary brain stem injury is diffuse axonal injury which affects the rostral dorsolateral aspect of the mid brain and upper pons. Commonly lesions are in the peril aqueduct region in comparison with a secondary lesion which usually appears in the ventral part of the brain stem (7).

On cranial CT scanning brain stem lesion are difficult to detect because of bone artifacts within the lower portion of posterior fossa. MRI is absolutely the method of choice in brain stem injury analysis and is also valuable in predicting the outcome $(7,25)$.

\section{Secondary injuries}

The secondary effect of a craniocerebral trauma is sometimes of greater importance than direct manifestation (7). Secondary alterations include, diffuse cerebral edema, Hypoxia, infarction, necrosis, secondary hemorrhage and cerebral herniation which is of types subalpine, transtentorial and tonsil herniation. Most secondary injuries are caused by an increased intracranial pressure or cerebral herniation $(7,11)$.

Using CT and MRI those lesions can be easily distinguished from the late squeal which is the final result of brain injury such as hydrocephalus, pneumocephalus, CSF leak, and encephalomalacia (7).

\section{Methodology}

\section{Patients and methods}

This prospective study was done in the spectated surgical hospital and neurological hospital from October-2016 to July 2017 on 160 patients whose ages ranged from 21-64 years, presented in emergency department with acute head trauma and the first CT done for them had been selected and radio logically analyzed.

*All CT scan were taken within the first 24 hours of injury.

*All patients had full physical and neurological examination.

*The CT findings were intercepted by consultant radiologist.

*The examination of all patients was performed by spiral CT soma ton plus4 unit (S Siemens medical system).

*All patients were examined in supine position without intravenous contrast media.

\section{CT Protocol}

1. Slice thickness 5-10 mm

2. Kilo Voltage (KVP) $12 \mathrm{KV}$

3. Milliamp second per slice 200-300 mass

4. Brain window Width 80

LEVEL 40

5. Bone Window Width 2500

Level 250

6. Table increment 1.50 


\section{CT scanning technique}

In CT scan for patients with acute head trauma, patient was scanned in supine position with or without sedation or anesthesia. The scan extended from the foramen magnum to the vertex and plane of section angled parallel to the line between the floor of frontal fossa and the foramen magnum to obtain true axial images. A compromise was made between slice thickness, the signal-to-noise ratio and contrast solution. Contiguous $10 \mathrm{~mm}$ section was usually used to exclude neurosurgical significant hematoma. In the posterior fossa the slice thickness did not exceed $5 \mathrm{~mm}$ in order to minimize partial volume averaging which can obscure small but potentially significant hematoma and contusion. Soft tissue (brain) and bone window images were obtained from the same data set and 3-D reconstruction were interpreted when they were available $(13,14)$.

\section{RESULTS AND DISCUSSION}

\section{Results}

In this prospective study the number of the patients was 160, 106 (66\%) males and 54 (34\%) females with male to female ratio $2 / 1$, the commonest

TABLE 1. Age and gender distribution

\begin{tabular}{|c|c|c|c|c|}
\hline \multirow{2}{*}{ Age (Yr.) } & \multirow{2}{*}{ Male No. } & \multirow{2}{*}{ Female No. } & \multicolumn{2}{|c|}{ Total } \\
\cline { 3 - 5 } & & & No. & $\%$ \\
\hline $20-29$ & 50 & 22 & 72 & $45 \%$ \\
$30-30$ & 24 & 16 & 40 & $25 \%$ \\
$40-49$ & 18 & 6 & 24 & $15 \%$ \\
$50-59$ & 10 & 8 & 18 & $11 \%$ \\
$60-69$ & 4 & 2 & 6 & $4 \%$ \\
\hline Total & 106 & 54 & 60 & $100 \%$ \\
\hline
\end{tabular}

age group was between 20-29 years as shown in (Table 1 and Fig. 1).

Patient with blunt (closed) head injury were 16 (10\%), while 34 patients $(21.25 \%)$ had penetrating (open) head injury.

The main indications for CT as shown in (Table 2 and Fig. 2) are in order of frequency, disturbances in the level of consciousness as found in 62 patients (38.75\%), followed by multiple injuries including thoracic and abdominal injuries seen in 34 patients (21.2), penetrating head injury seen in 31 patients (19.3\%). Progressive headache after head injury in 30 patients $(18.7 \%)$,vomiting more than one episode seen in 17 patients $(10.6 \%)$, and only 6 patients $(3.7 \%)$ presented with signs of fracture of skull base including CSF rhinorrhea, otorrhea ,cranial nerve dysfunction, battle sign (Mastoid) and Raccoon (black) eye. One hundred twenty patients (75\%) had abnormal CT finding while 40 (25\%) had normal CT as shown in (Table 3 and Fig. 3).

The incidence of CT abnormalities according to the mechanism of injury is demonstrated in (Table 3 and Fig. 3), which shows that the diffuse swelling of the entire brain takes place mainly in adolescents \& children. It is several types in head injury, the commonest is hyperemic brain swelling, and here the brain swells due to an increase in blood volume, not edema. Grey-white matter contrast is preserved on both CT and MRI and it can be difficult to assess. Diffuse swelling of the ipsilaterl hemisphere may also happen with focal and mass lesions, including acute subdural hematoma and extensive unilateral contusion.

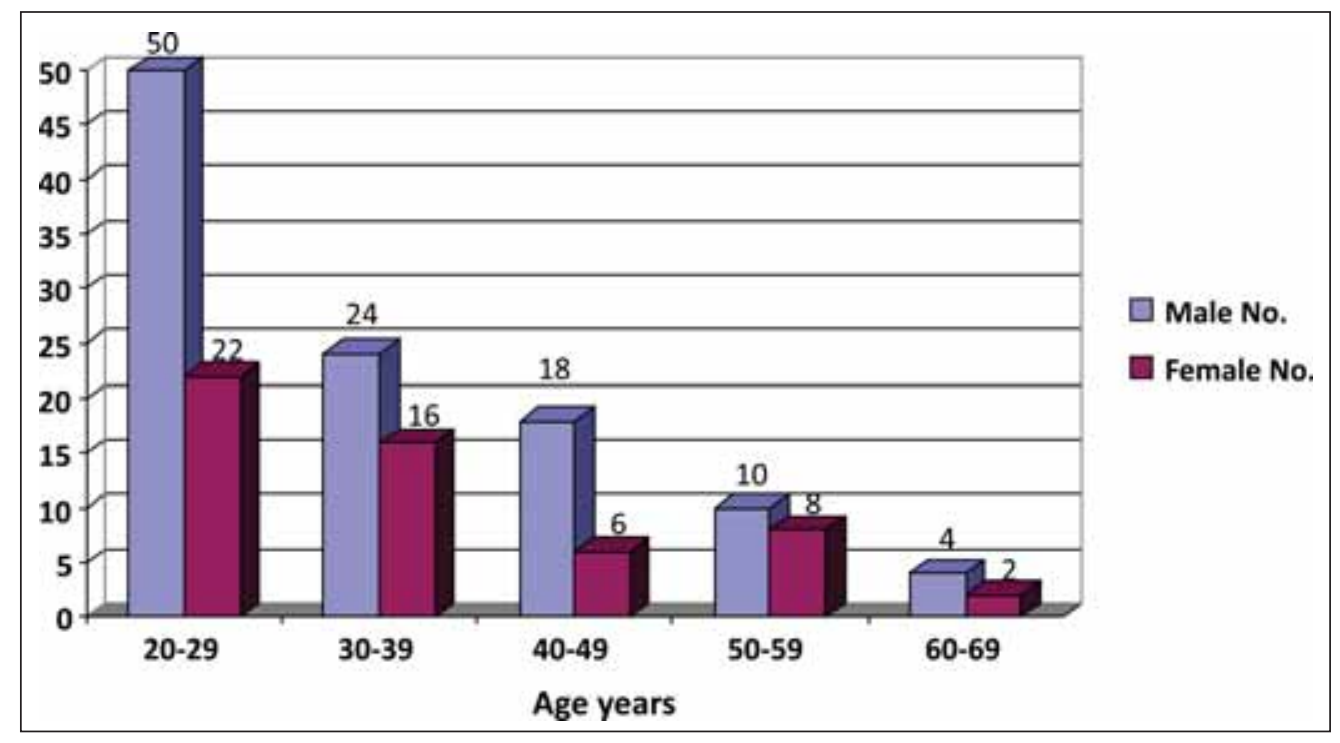

FIGURE 1. Age and gender distribution 
TABLE 2. Distribution of patient according to their indications for brain CT.

\begin{tabular}{|l|c|c|}
\hline Indication & No. of Patients & $\%$ \\
\hline Disturbances in level of consciousness & 62 & $38.75 \%$ \\
Mul ple injuries & 34 & $21 \%$ \\
Penetra ng head injury & 31 & $19.3 \%$ \\
Progressive headache & 30 & $18.7 \%$ \\
Vomi ng & 17 & $10.6 \%$ \\
Fracture of skull base & 6 & $3.7 \%$ \\
\hline
\end{tabular}

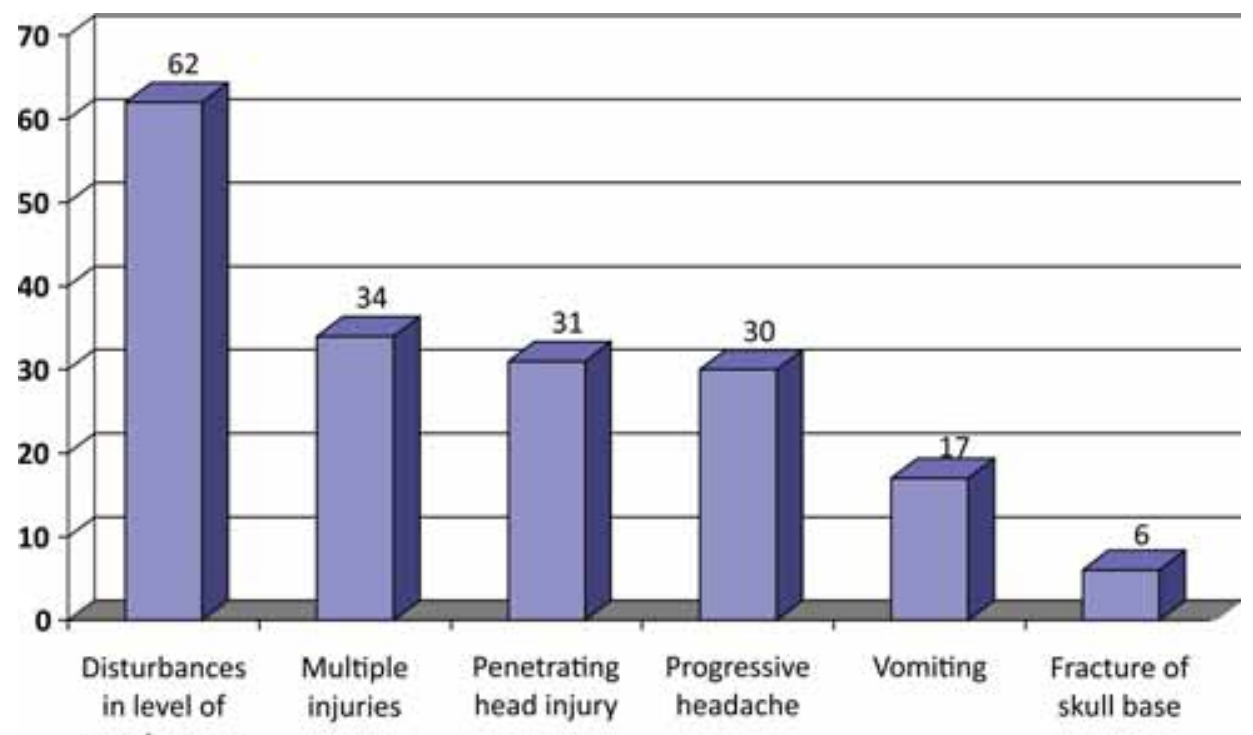

Indication

FIGURE 2. Distribution of patient according to their indications for brain CT.

TABLE 3. Incidence of CT abnormalities according to mechanism of injury

\begin{tabular}{|l|c|c|c|c|c|c|}
\hline \multirow{2}{*}{ Mechanism of injury } & \multicolumn{2}{|c|}{ Abnormal of CT } & Normal CT & & \multicolumn{2}{|c|}{ Total } \\
\cline { 2 - 7 } & No. & $\%$ & No. & $\%$ & No. & $\%$ \\
\hline Road traffic accident & 46 & $28.75 \%$ & 17 & $26.9 \%$ & 63 & $39.4 \%$ \\
Penetra ng & 34 & $21.25 \%$ & - & - & 34 & $21.2 \%$ \\
FFH & 20 & $12.5 \%$ & 8 & $28.5 \%$ & 28 & 17.5 \\
Blunt injury of blast & 16 & $10 \%$ & 9 & $36 \%$ & 25 & $15 \%$ \\
Assaults & 4 & $02.5 \%$ & 6 & $60 \%$ & 10 & $6.2 \%$ \\
\hline Total & 120 & $75 \%$ & 40 & $25 \%$ & 160 & $100 \%$ \\
\hline
\end{tabular}

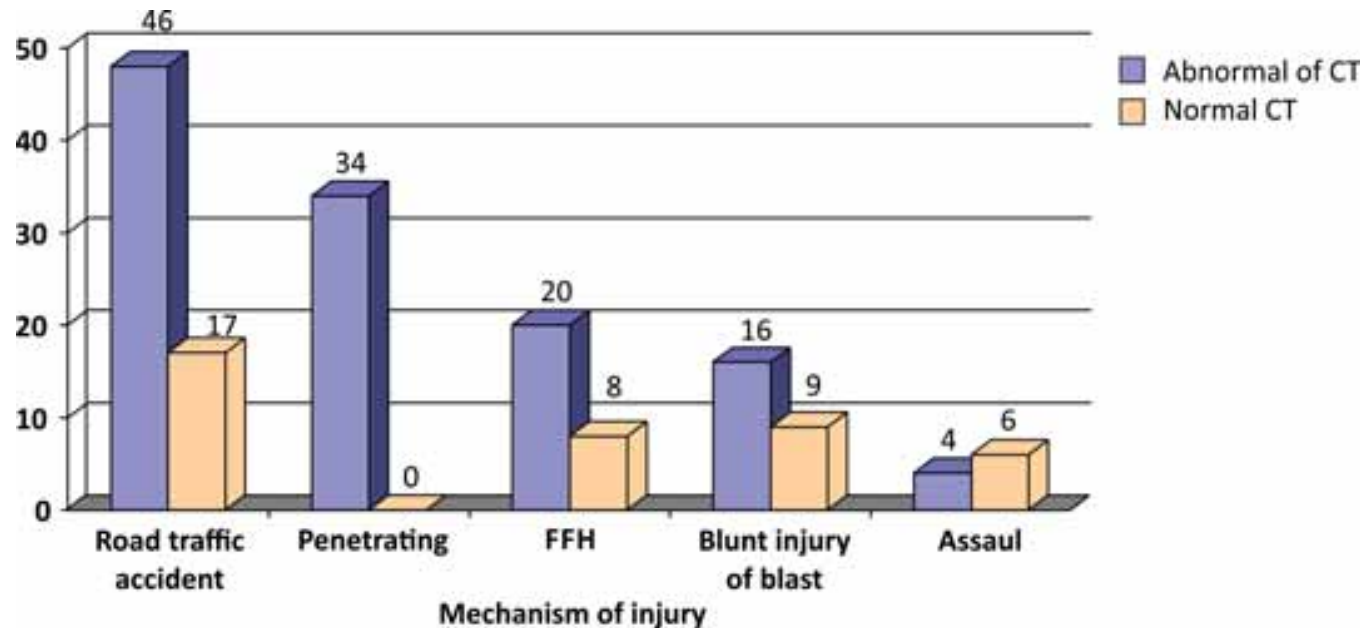

FIGURE 3. Incidence of CT abnormalities according to mechanism of injury 
With diffuse bilateral swelling, the ventricles appear as small, slit-like and compressed at imaging; the basal iterus may be occluded and the sulcieffced cerebellum may appear relatively hyper dense in comparison with the edematous cerebral hemispheres (white cerebellum high). In the early stage, the white matter shows density reduction and appears normal at $\mathrm{CT}$, despite the evidence of edema which may be seen in cases with prolonged coma.

\section{Hypoxic brain damage}

This is due to ether prolonged low systolic blood pressure, or to arterial spasm or to both. Hypoxia \& subsequent infarction can also be the result of brain swelling and tectorial herniation which compromise the posterior cerebral circulation or from trauma to motor vessels. Little may be shown by imaging in the early stages, but evidence of infarction will be demonstrated later.

The road traffic accident ( RTA) is the most common mechanism of head injury seen in 63 patients (39.4\%) and, $46(73.1 \%)$ of them had abnormal CT findings while 17 (10.62\%) had normal CT, the second most common mechanism was penetrating injury seen in 34 patients $(21.25 \%)$, all of them had abnormal CT, while fall from height (FFH) was the cause in 28 patient $(17.5 \%), 20(71.5 \%)$ of them had abnormal CT, while 8 (5\%) had normal CT, blunt injury of blast was the mechanism of head injury in 25 patients $(15.7 \%), 16(10 \%)$ of them had abnormal CT while 9 (5.62\%) had normal CT, the least common mechanism was assault seen in 10 patients $(6.2 \%), 4(2.5 \%)$ of them had abnormal CT while $6(3.75 \%)$ had normal CT .Acute head trauma was divided into 3 types according to the Glasgow coma scale (GCS) as shown in (Table 4 and Fig. 4) including: moderate head trauma with GCS (9-12) seen in 60 patients $(37.5 \%)$ was the most common type, $48(30.1 \%)$ of them had abnormal CT while 12 (7.5\%) had normal CT. mild head trauma with GCS (13-15) seen 53 patients (33.2\%), 33 (20.6\%) of them had abnormal CT while $20(12.5 \%)$ had normal CT. forty seven patients $(29.3 \%)$ presented with severe head injury with GCS $(<8), 39$ patients $(24.3 \%)$ of them had abnormal CT and only 8 patients $(5 \%)$ had normal CT. Skull fracture was the commonest abnormal CT finding seen in 70 patient $(43.7 \%)$, most commonly in the parietal region of the head as shown in (Tables 5, Fig. 5 and (Table 6, Fig. 6), followed in order of frequency by: cerebral contusion seen in 47 patients $(29.3 \%)$ most commonly in Frontal region of the brain, subgalial hematoma seen in 34 patients $(21.2 \%)$, intracranial foreign body seen in 30 patients $(18.7 \%)$, intracranial air in 27 patients $(16.8 \%)$ most commonly seen

TABLE 4. Incidence of CT abnormalities according to GCS

\begin{tabular}{|l|c|c|c|c|c|c|}
\hline \multirow{2}{*}{ Type of trauma according to GCS } & \multicolumn{2}{|c|}{ Abnormal CT } & \multicolumn{2}{c|}{ Normal CT } & \multicolumn{2}{c|}{ Total } \\
\cline { 2 - 7 } & No. & $\%$ & No. & $\%$ & No. & $\%$ \\
\hline Mild (13-14) & 33 & $20.6 \%$ & 20 & $12.5 \%$ & 53 & $33.2 \%$ \\
Moderate (9-12) & 48 & $30.1 \%$ & 12 & $7.5 \%$ & 60 & $37.5 \%$ \\
Sever (8) & 39 & $24.3 \%$ & 8 & $5 \%$ & 47 & $29.3 \%$ \\
\hline Total & 120 & $75 \%$ & 40 & $25 \%$ & 160 & $100 \%$ \\
\hline
\end{tabular}

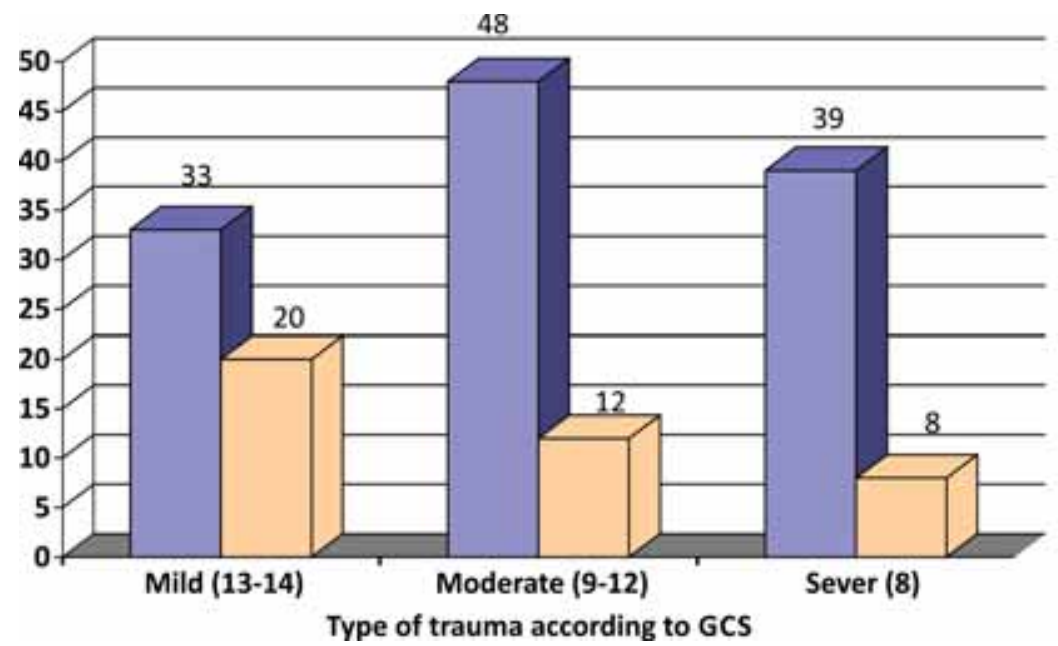


TABLE 5. CT Findings in 160 Patients

\begin{tabular}{|l|c|c|}
\hline CT finding & No. of patients & $\%$ \\
\hline Skull fracture & 70 & $43 \%$ \\
Cerebral Contusion & 47 & $29.3 \%$ \\
Normal & 40 & $25 \%$ \\
Subgalial hematoma & 34 & $21.2 \%$ \\
Intracranial foreign body & 30 & $18.7 \%$ \\
Intracranial air & 27 & $16.8 \%$ \\
Epidural hematoma & 15 & $9.3 \%$ \\
Subdural Hematoma & 12 & $7.5 \%$ \\
Subarachnoid hematoma & 11 & $6.8 \%$ \\
Diffuse cerebral edema & 10 & $6.2 \%$ \\
Intraventricular hemorrhage & 8 & $5 \%$ \\
\hline
\end{tabular}

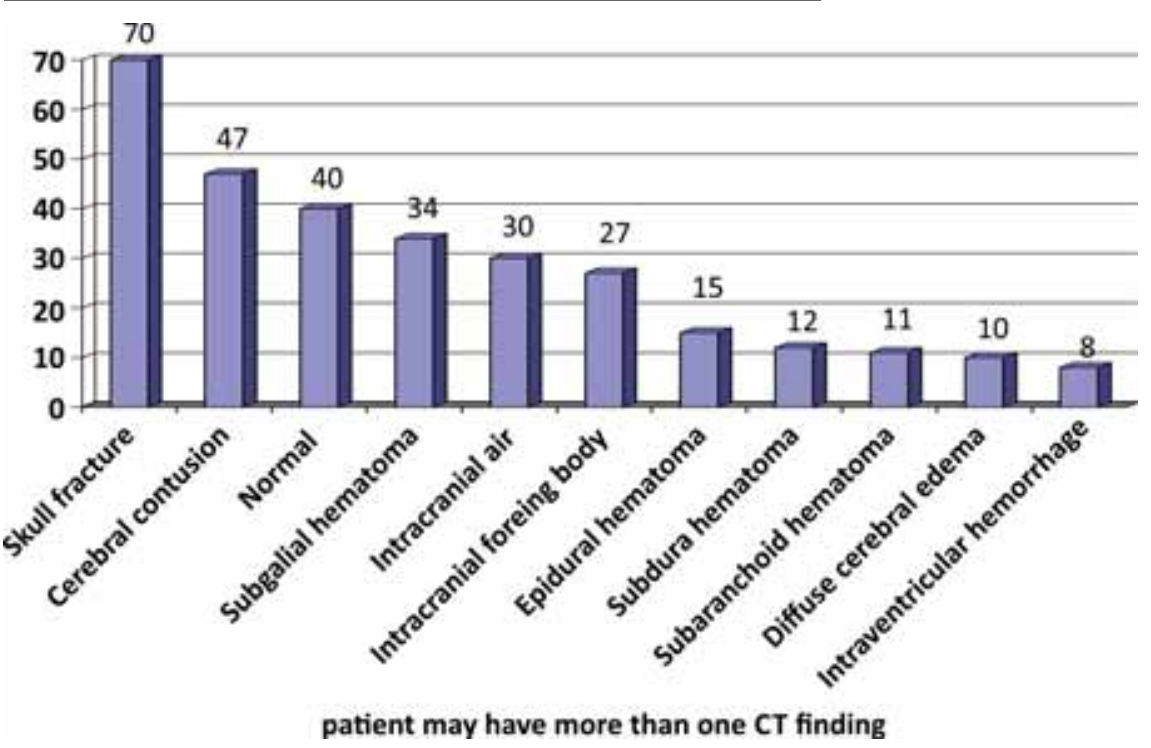

FIGURE 5. CT Findings in 160 Patients

in parietal region, epidural hematoma seen in 15 patients $(9.3 \%)$ most commonly in parietal region, 12 patients of study $(7.5 \%)$ had subdural hematoma which was most commonly seen in parietal region of the brain, subarachnoid hemorrhage seen in 11 patients $(6.8 \%)$, diffuse cerebral edema seen in 10 patients $(6.2 \%)$ and only 8 patients $(5 \%)$ had intraventricular hemorrhage. Among the patient with skull fracture, the linear fracture was the commonest type seen in 33 patients $(47.2 \%)$, followed by comminuted fracture in 20 patients $(28.6 \%)$, and 17 patients $(24.2 \%)$ had depressed fracture.

In 15 patients with epidural hematoma, 14 patients $(93.3 \%)$ of them were associated with skull fracture, while only one patient $(6.7 \%)$ had epidural hematoma without skull fracture, and in 12 patients with subdural hematoma's 9 patients $(75 \%)$ were associated with skull fracture, while 3 patients (25\%) had subdural hematoma's without skull fracture. so association with skull fracture was more in epidural than subdural hematoma's. Eighty-eight patients (55\%) had skull x-ray, $55(62.5 \%)$ of them were normal, 19 patients $(21.5 \%)$ had linear frac- ture, 8 patients $(9.2 \%)$ had comminuted fracture and only 6 patients $(6.8 \%)$ had depressed fracture (Table 7 \& Fig. 7).

\section{DISCUSSION}

Head trauma is a main problem in accident and emergency department. In the United Kingdom, it is responsible for 150000 hospital admissions yearly (17).

In this study the closed head injury was the commonest type of head injury, presented in $78.75 \%$ of patients, while only $21.25 \%$ of patients had open head injury, this is comparable to the results reported by Dickson's et al (1999). (8)Also the ratio of male to female was 2:1 and the commonest affected age group was between 20-29 years, as the males were approximately twice as likely as females to sustain traumatic brain injury, with peak age of incidence was 18-30 years This is in agreement with other studies (5).

On examination 120 patients (75\%) had abnormal CT findings while 40 (25\%) had normal CT 
TABLE 6. Distribution of abnormal CT findings in the head.

\begin{tabular}{|l|c|c|c|c|c|c|c|c|c|c|}
\hline \multirow{3}{*}{ Location } & \multicolumn{10}{|c|}{ CT finding } \\
\cline { 2 - 12 } & \multicolumn{1}{|c|}{ Skull fracture } & \multicolumn{2}{|c|}{ E.D.H } & \multicolumn{2}{c|}{ S.D.H } & \multicolumn{2}{c|}{ Cerebral contusion } & \multicolumn{2}{c|}{ Intracranial air } \\
\cline { 2 - 11 } & No. & $\%$ & No. & $\%$ & No. & $\%$ & No & $\%$ & No & $\%$ \\
\hline Frontal & 18 & $25.8 \%$ & 4 & $26.6 \%$ & 3 & $25 \%$ & 23 & $49 \%$ & 10 & $37.1 \%$ \\
Parietal & 28 & $40 \%$ & 9 & $60 \%$ & 5 & $41 \%$ & 14 & $29.8 \%$ & 12 & $44.4 \%$ \\
Temporal & 7 & $10 \%$ & 2 & $13.4 \%$ & 4 & $33.3 \%$ & 4 & $8.5 \%$ & 3 & $11.1 \%$ \\
Occipital & 9 & $12.8 \%$ & - & - & - & - & 6 & $12.7 \%$ & 2 & $7.4 \%$ \\
Base of skull & 8 & $11.4 \%$ & - & - & - & - & - & - & - & - \\
\hline Total & 70 & $100 \%$ & 15 & $100 \%$ & 12 & $100 \%$ & 47 & $100 \%$ & 27 & $100 \%$ \\
\hline
\end{tabular}

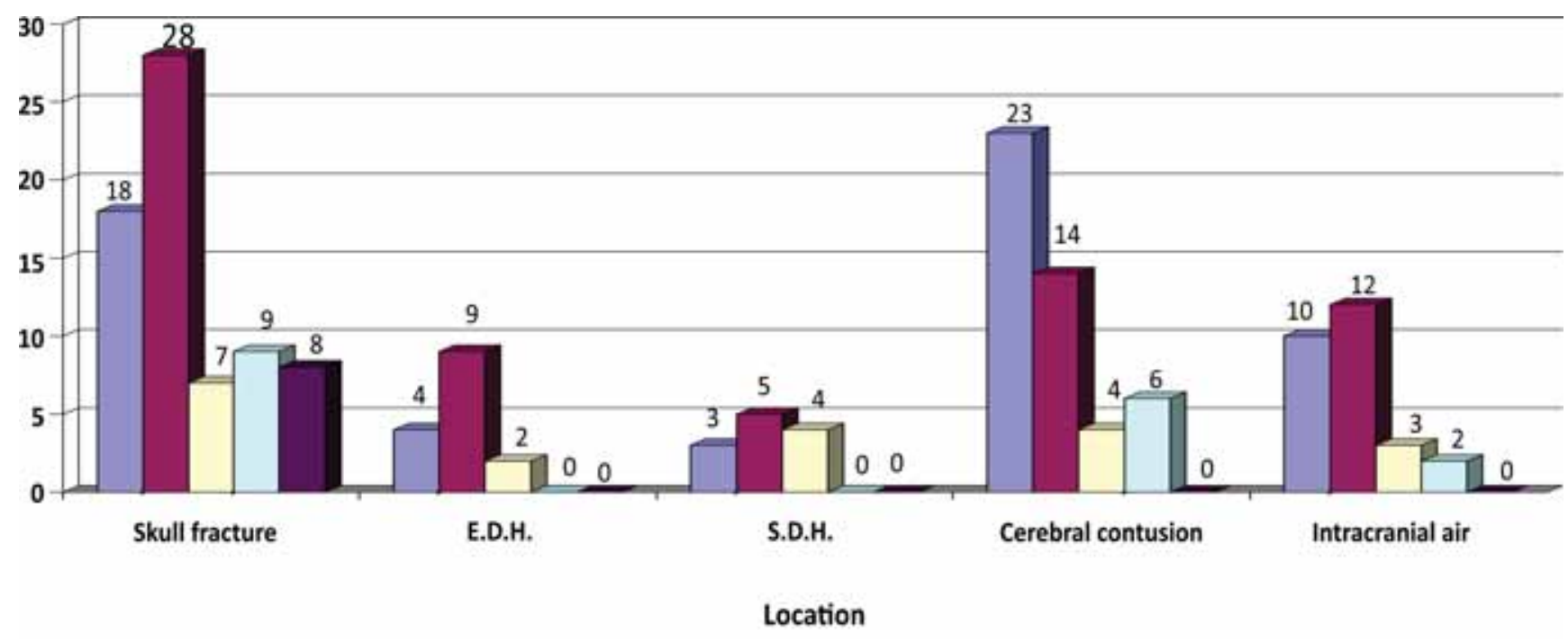

口 Frontal $\quad$ Parietal $\square$ Temporal $\square$ Occipital Base of skull

FIGURE 6. Distribution of abnormal CT findings in the head.

TABLE 7. Skull X-ray findings.

\begin{tabular}{|l|c|c|}
\hline X-ray findings & No.of patients & $\%$ \\
\hline Normal skull X-ray & 55 & $62.5 \%$ \\
\hline Linear fracture & 19 & $21.5 \%$ \\
\hline Comminuted fracture & 8 & $9.2 \%$ \\
\hline Depressed fracture & 6 & $6.8 \%$ \\
\hline Total & 88 & $100 \%$ \\
\hline
\end{tabular}

55

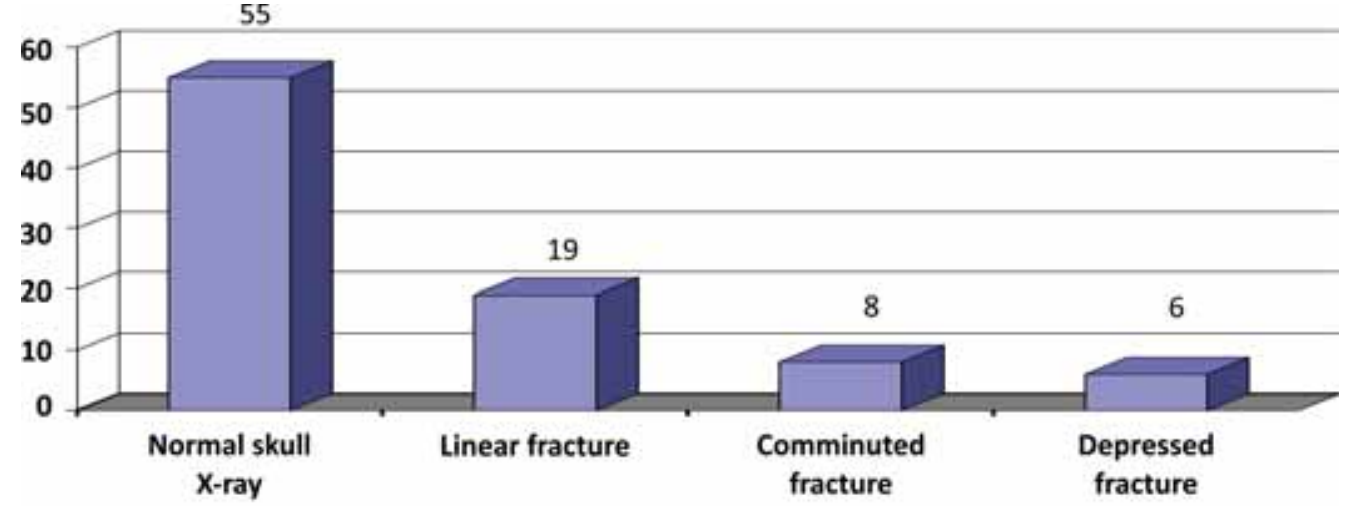

Skull $X$-ray findings

FIGURE 7. Skull X-ray findings 
findings, these results correlate with the results of Besenski (2012) (7), while does not correlate with the results of Maharjan S. et al (2017) (5) who reported that $75.1 \%$ of patients had normal CT findings and only $24.9 \%$ had abnormal CT findings. This miscorrelation of our results with the last study is due to selection bias and variations in mechanism of traumatic brain injury.

Road traffic accident (RTA) was the most common mechanism of head trauma seen in 63 patients (39.4\%) (Table 3). These results correlate with other studies Obajimi et al. (2002) in which all agree with RTA was the most common mechanism of traumatic brain injury account for approximately one half of the cases (Obajimi et al., 2002).

But the results in our study do not correlate with the results Of Maharjan S.et al. (2017) (5) who reported that fall from height $(\mathrm{FFH})$ was the most common mechanism of head trauma $(71.6 \%)$ followed by RTA (22.4\%) this is because of selection bias.

According to results of studies by Maharjan S. et al (2017) (5), Dickson et al (1999) (8) and segue (2005) (6) where Glasgow coma scale (GCS) points used to categorize head trauma severity, mild head injury was the commonest type followed by moderate head injury and severe head injury, while Novak (2000) (4) reported that severe head injury was the commonest type seen in $58.8 \%$ of patients, followed by mild head injury seen in $21.2 \%$ of patients and only $20 \%$ of patients had moderate head injury. in contrast, results of this study shows that moderate head trauma (score 9-12) was the most common type (Table 4) and this disagreement is probably attributable to the selection bias and differences in the cause of traumatic brain injury.

Also we found that skull fracture was the commonest abnormal CT finding seen in 70 patients (43.7\%), followed by cerebral contusion seen in 47 patients $(29.3 \%)$ as the commonest focal lesion in the brain (Table 5).these results are correlated well with the results of Besenski (2002) (7) and souse et al (2002) (27), but are in contrast to the result of Alisa (2003) (5) in which the commonest focal lesion in the brain following of our results with the last study is most probably because of differences in mechanism of injury. In this study, 88 patients (55\%) had skull x-ray, $55(62.5 \%)$ of them were normal and 33 patients (37.5\%) of them had abnor- mal skull x-ray findings (Table 7), while Segun T. (2005) (6) reported that (72.2\%) of patients had skull x-ray, $(84.9 \%)$ of them were normal and the most common abnormal skull $x$-ray finding, as in the current study, was the linear skull fracture. These differences in results most probably because of variations in the mechanism of injury and severity of traumatic brain injury in emergency department.

\section{Skull fracture}

CT scan detects skull fracture in approximately two-third of patients with acute head trauma, skull fractures include: linear, depressed, diastolic, comminuted and compound types or combinations and the linear fracture is the most common type $(8,10)$.

In this study, skull fractures were the commonest abnormal CT finding seen in 70 patients (43.7\%), linear fracture was the commonest type seen in 33 patients $(47.2 \%)$, followed by comminuted fracture in 20 patients $(28.6 \%)$ and 17 patients $(24.2 \%)$ had depressed fracture. Gul Muhammad et al (2017) (10).

\section{Cerebral contusion}

In CT scan cerebral contusion is the most common acute traumatic brain lesion. The frontal and temporal lobes are the classic sites of cerebral contusion $(23,24)$.

In the current study, the Cerebral contusions were the commonest focal lesion in the CT scan seen in 47 patients $(29.3 \%)$ most commonly in the frontal region $(49 \%)$, followed in order frequency by parietal region $(29.8 \%)$, occipital region (12.7\%), and temporal region (8.5\%).

\section{Epidural hematoma}

In cases of acute traumatic brain injury, Epidural hematoma's are identified by CT scan in $(8.3 \%)$ of cases, most frequency in the front parietal region and associated with skull fracture in approximately (95\%) of cases $(5,25)$.

In this study, Epidural hematoma's were seen as abnormal CT finding in 15 patients (9.3\%), most frequently in parietal region $(60 \%)$, followed by frontal region $(26.6 \%)$ and temporal region (13.4\%).most of these Epidural hematoma's were associated with skull fracture (93.3\%), while (6.7\%) were without skull fractures. 


\section{Subdural hematoma}

A subdural hematoma demonstrated by CT.

It is important that a person receive medical assessment, including a complete neurological examination, after any head trauma. A CT scan or MRI scan will usually detect significant subdural hematomas. Subdural hematomas occur most often around the tops and sides of the frontal and parietal lobes $(3,8)$. They also occur in the posterior cranial fossa, and near the falx cerebral and tentorium cerebella (3). Unlike epidural hematomas, which cannot expand past the sutures of the skull, subdural hematomas can expand along the inside of the skull, creating a concave shape that follows the curve of the brain, stopping only at the Dural reflections like the tentorium cerebella and falx cerebra.

On a CT scan, subdural hematomas are classically crescent-shaped, with a concave surface away from the skull. However, they can have a convex appearance, especially in the early stage of bleeding. This may cause difficulty in distinguishing between subdural and epidural hemorrhages. A more reliable indicator of subdural hemorrhage is its involvement of a larger portion of the cerebral hemisphere since it can cross suture lines, unlike an epidural hemorrhage. Subdural blood can also be seen as a layering density along the tentorium cerebella. This can be a chronic, stable process, since the feeding system is low-pressure. In such cases, subtle signs of bleeding such as effacement of sulci or medial displacement of the junction between gray matter and white matter may be apparent.

Fresh subdural bleeding is hyper dense, but becomes more hypo dense over time due to dissolution of cellular elements. After somewhere between 3-14 days, the bleeding becomes is Odense with brain tissue and may therefore be missed (18). Subsequently, it will become more hypo dense than brain tissue By CT scan subdural hematomas are seen in $(4.8 \%)$ of cases of acute head trauma, most of such hematomas are located in the front parietal areas or middle cranial fossa.

Skull fractures are less commonly present in patients with acute subdural hematomas than epidural bleeds $(7,10)$.

In our study, subdural hematoma's were seen in CT scan in 12 patients (7.5\%), most commonly in the parietal region $(41.7 \%)$, followed by temporal region $(33.3 \%)$ and $(25 \%)$ was in frontal region.

Skull fractures were less commonly present in patients with acute subdural hematoma's than epidural hematoma's, as (93.3\%) of epidural hematoma's were associated with Skull fracture while (75\%) of subdural hematoma's associated with Skull fracture.

\section{Intraventricular hemorrhage}

In CT scan the Intraventricular hemorrhage is generally found in fewer than $5 \%$ of patients with acute traumatic brain injury (10). Intraventricular hemorrhage was detected in only $8(5 \%)$ of patients in this study.

\section{CONCLUSION}

CT is the preferred imaging technique for a patient with a head injury and may require surgical intervention. They are quick, accurate and easily available in most cases where there is an emergency medical facility. Radiation tomography involves the use of ionizing radiation. It is widely accepted that patients who do not have mild head injuries should receive CT scans. For those with mild head injuries, CT scans should only be referred to those patients who have been identified through systematic clinical evaluation as being at increased risk of intracranial injuries (27). When CT is abnormal in a patient with mild head injury, abnormalities usually do not require specific treatment. Injuries such as unbroken skull fractures, small brain hemorrhages (or bruising) or bleeding outside the brain (subcutaneous hemorrhage and subcutaneous hemorrhage) are the most common results in the minority of patients with mild head injuries and abnormal CT scan.

CT scan is still the primary method for evaluating patients with severe head trauma (26). It is fast, easy and reliable, and is the best option to show a skull fracture or severe bleeding inside axial or axial outside. Increased incidence of abnormal CT results with increased severity of head trauma.

Many people believe that MRI, as a newer and more expensive imaging technique, should be better than CT. This idea is incorrect, because each has its own use. CT scans are faster than MRI, so they are good in emergency situations. CT is better than 
magnetic resonance imaging to look at the bones and detect new bleeding in or around the brain. For these reasons, CT is the preferred imaging technique for assessing head trauma (2). Traditional radiography has a small role in evaluating patients with severe head injuries. Its use has declined significantly since the advent of the CT scanner, and skull films are no longer widely used except in rare cases where CT scans were not available and the main indication for CT scan was disturbance in the level of consciousness. Skull films are not sensitive and specific to detect intracranial injuries, although a skull fracture dose increases the likelihood of intracranial dysfunction, nearly half of all cranial abnormalities resulting from head trauma have no skull fracture associated with them, so absence does not reduce A skull fracture in the X-ray skull requires a scanner, because it is more sensitive to soft tissue injuries, superior to spatial localization and evaluation of bone changes.

Patients with the affected head must be assessed through a structured clinical assessment process involving a combination of obtaining a precise history of disease presentation, physical and neurological examination, and clinical clinical observations. Sequential evaluation is vital because the patient may rapidly deteriorate due to bloody swelling or swelling. The assessment should determine whether the patient has a high or low risk of significant brain injury.

Conflict of interest: none declared Financial support: none declared

\section{REFERENCES}

1. Sir Godfrey Newbold Hounsfield, CBE, FRS, (1919-2004) was an English electrical engineer who shared the 1979 Nobel Prize for Physiology or Medicine with Allan McLeod Cormack for his part in developing the diagnostic technique of X-ray computed tomography (CT). His name is immortalised in...

2. Jonathan M. Ford, Summer J. Decker 2005. Computed Tomography slicethickness and its effecton three dimension reconstructions of anatomical structures, Journal of Forensic Radiology and imaging, Vol.4; page 43-46.

3. Jennifer Beecham, Margaret Perkins, Tom Snell \& Martin Knapp, 2009 Treatment paths and costs for young adults with acquired brain injury in the United Kingdom, Journal Brain Injury, Pages 30-38

4. Simon Shorvon, Renzo Guerrini, Mark Cookand samden D. Lhatoo, (2012), Oxford Textbook in clinical Neorology of Epilepsy and Epileptic Seizures, Oxford university Press 2013.

5. Alan Felthous M.D., Henning SaB M.D. (2008) - Psychology, The International Handbook of Psychopathic Disorders ... Print ISBN:9780470066386 |Online ISBN:9780470772973 |DOI:10.1002/9780470772973

6. Segun T. (2005) Traumatic brain injury: definition, epidemiology, pathophysiology. Emerg Med; 9: 25-32.

7. Besenski N. (2002); Traumatic injuries: imaging of head injuries. Eur Radiology 12(6) 1237-1252.

8. Dickson S.C., Morris Kh. (1999) Evaluation of the patient with closed head trauma. Emerg Med Clin North Amer; 17: 15-25.

9. Bethesda M. (2003) Traumatic brain. Nucleus Med Art; 9: 30-33.

10. Mohamad Ghazali Masuria, Khairil Anuar Md Isab \& Mohd Pozi Mohd Tahirc, (2010), Children, Youth and Road Environment:Road Traffic Accident, Asia Pacific International Conference on Environment-Behaviour Studies, 38 (2012) 213-218.

11. Ann K. Kim MD, Laurie A. Loevner MD (2011), in Radiology Secrets Plus (Third Edition), Pages 327-335

12. Ralph W., Jack W., Mukesh H., John Chen, (2011) Primer of diagnostic imaging, 5th ed. Page Count: 816

13. Zee C.S., Go J.L., (1998) CT of head trauma. Neuro imaging Clin N Am; 8(3): 525-539.

14. Mittl R.L., Grossman R.I., Hiehle J.F., Hurst R.W., Kauder D.R., Gennarelli T.A., Alburger G.W. (1994). Prevalence of MR evidence

of diffuse axonal injury in patients with mild head injury and normal head CT findings. AJNR Am J Neuroradiology; 15(8): 1583-1589.

15. Gentry L.R., Godersky J.C., Thompson B., (1994) MR imaging of head trauma: Review of the distribution and radiopathologic features of traumatic lesions. AJR Am J Roentgenol. 150(3):663-672

16. Gardner, R. Zafonte, (2016) in Handbook of clinical Neurology..

17. Sutton D. (2002) Textbook of radiology and imaging, 7 ed., vol. 2. London: Churchill living stone, 1641-1642, 1779-1782.

18. Kreitzer N., Lyons M.S., Hart K., Lindsell C.J., Chung S., Yick A., Bonomo J. (2014) Repeat neuroimaging of mild traumatic brain-injured patients with acute traumatic intracranial hemorrhage: clinical outcomes and radiographic features. Acad Emerg Med. 21(10):1083-1091

19. Pitts L.H. (1991) the role of Neuroimaging in minor head trauma. Ann Emerg Med; 20 (12): 1387-1388.

20. Mohanty S.K.,Thompson W., Rakower W. (1991) are the CT scan for head injury patients always necessary? Trauma; 31(6):804-805.

21. James E., Gillespie (Editor), Jackson A, (2000), MRI and CT of the brain Book. London: Arnold, 1 ed.; 73-90.

22. Akinwunmi Olalekan Akanji, Rachael Adeyanju Akinola, Babajide Olawale Balogun, Aliu Olabanji Akano, Omolola Mojisola Atalabi, Michael A. NAkinkunmi, Gbolahan O.G. Awosanya, (2015), Computerized tomography scan and head injury.Vol.6(1)PP,1-15.

23. Sousa J., Sharma R.R., Pawar S.J. (2002) Long term outcome in patients with head injury. Neurology india; 50: 430-435.

24. Cihangiroglu M., Mutlu R.G., Ramsey G.J., (2002). Brain injury: analysis of imaging modalities. Neurol. Research; 24(1): 7-18.

25. Zimmerman R.A., Bilaniuk L.T., Gennarelli T. (1978). Cranial computed tomography in diagnosis and management of acute head trauma AJR Volume 131, Number (1) 1311:27-32.

26. Kido D.K., Cox, Hamill R.W., (1992). Traumatic brain injuries: predictive usefulness of CT. Radiology; Department of Radiology, University of Rochester Medical Center vol.182: 777-781.

27. Reed D. Adult Trauma Clinical Practice Guidelines, Initial Management of Closed Head Injury in Adults. 2nd ed: NSW Institute of Trauma and Injury Management; 2011. 